\title{
GENERATION OF PICOSECOND X-RAY PULSES IN THE ALS USING RF ORBIT DEFLECTION*
}

\author{
David Robin, John Byrd, Peter Fischer, Phil Heimann, Dong-Hyun Kim, Slawomir Kwiatkowski, \\ Derun Li, Fernando Sannibale, Christoph Steier, Weishi Wan, Walter Wittmer, Alexander Zholents, \\ LBNL, Berkeley, CA 94720, U.S.
}

\begin{abstract}
A scheme is proposed for producing ps length pulses of $\mathrm{x}$ ray radiation from the Advanced Light Source (ALS) using two RF deflecting cavities. The cavities create vertical displacements of electrons correlated with their longitudinal position in the bunch. The two cavities separated by 180 degrees of vertical phase advance. This allows the vertical kick from one cavity to be compensated by the vertical kick of the other. The location of the cavities corresponds to the end of one straight section and the beginning of the following straight section. Halfway between the cavities a bending magnet source is located. The radiation from the bend can be compressed to $\sim 1$ ps in duration.
\end{abstract}

\section{INTRODUCTION}

A synchrotron light source, such as the ALS, can produce high average brightness radiation from infrared to the hard $\mathrm{x}$-rays. One of the nice features of a synchrotron light source is that the light is emitted in pulses rather than continuously in time. Electron bunches circulating in a storage ring produce synchrotron light in pulses with a typical FWHM duration of a hundred picoseconds defined by the electron bunch length. Experiments can then exploit the time structure to study the dynamics of various phenomena.

Although the present 70 ps time duration is adequate for some applications $[6,7]$, there is a strong demand for many new exciting scientific opportunities utilizing X-ray pulses at time scales down to the ps range [8].. We are proposing a technique (the picosecond source) that would reduce the light pulse length 2 orders of magnitude over the present $70 \mathrm{ps}$ in normal ALS operation and possibly 3 orders of magnitude in special running conditions. An alternative approach to the picosecond source for generating short X-ray pulses is the femtoslicing technique [1] and is already in operation at the ALS. The picosecond and the femtoslicing techniques are complementary. The ultimate time resolution achievable with the picosecond technique during normal ALS operation is longer than that of slicing $(0.1 \mathrm{ps})$. There are certain applications that require time structure of less than 0.5 ps. However the flux of the picosecond source would be significantly higher than slicing due to the higher pulse repetition rate $(500 \mathrm{MHz}$ versus $20 \mathrm{kHz})$ and the higher per pulse flux. There is interesting science that would be enabled with picosecond time resolution such as the study of ultrafast magnetisation dynamics on a short (<10nm) length scale.

The picosecond source is included in the long range ALS strategic. In addition other laboratories are interested in providing a source of high repetition rate light pulses with a picosecond time duration. At the 2005 Users' Meeting for the Advanced Photon Source (APS) and the Center for Nanoscale Materials in May 2005, there was a workshop entitled "Generation and Use of Short X-ray Pulses at APS and there several papers being presented at this meeting $[2,3,4]$.

\section{SCIENTIFIC MOTIVATION - ULTRAFAST MAGNETISM DYNAMICS}

Precessional frequencies of the magnetization in nanostructured ferromagnetic elements increase with decreasing length size. The current lateral resolution of soft X-ray microscopy $(15 \mathrm{~nm})$ is able to record full-field images of magnetic domain structures at the granular length scale by utilizing X-ray magnetic circular dichroism as element specific (e.g. layer resolved) magnetic contrast mechanism. The current stroboscopic pump-and-probe setup at XM-1 allows us to study e.g. vortex dynamics in elements of several micron size length scale with a time resolution of $70 \mathrm{ps,} \mathrm{which} \mathrm{is} \mathrm{limited} \mathrm{by}$ the bunch structure of the ALS. However, ultrafast spin dynamics in elements of that size are relevant e.g. to understand nanomagnetism switching cannot be addressed. Improving the time resolution gradually down to the few ps regime would open a new area in combining high lateral and temporal resolution which is of high relevance both for fundamental studies of micromagnetism and particularly for technical applications in magnetic ultrafast high density storage and sensor developments. A real-space high resolution and ultrafast imaging technique with a field of view of several tens of micron provides valuable and direct information on correlated and coupled magnetisation phenomena.

Examples for typical studies/questions that can be addressed

- magnetization reversal processes of individual grains and large arrays of grains

- tailoring magnetic damping in confined nanosized geometries

- impact of local inhomogeneities, defects, shape fluctuations, etc. to magnetisation dynamics thermomagnetic writing in heat assisted magnetic recording schemes 
Requirements to perform these studies:

A) Source

- circular polarization (>10-20\%) for photon energies covering L-edges of $3 \mathrm{~d}$ elements and M-edges of $4 \mathrm{f}$ elements $(500-1200 \mathrm{eV})$ at reasonable photon flux (cf. 2-bunch mode operation)

- (tunable) time resolution down to $1 \mathrm{ps}$

B) Detector

- fast gateable CCD /2d-array detector allowing for multibunch mode operation /camshaft mode

C) Pump-Probe-Trigger scheme

- fast start signal (e.g. APD)

- $\quad$ Laser driven optical switches

- ultrafast magnetic field pulses to excite the sample's magnetization

\section{APPROACH}

It is not possible to significantly reduce the electron bunch length in a storage ring. Nevertheless it is possible to manipulate the electron beam to produce short light pulses. Here we consider a technique where pulses of synchrotron radiation as short as a picosecond can be obtained without a reduction of the bunch length. We propose to do this by creating a condition where the radiation of electrons sitting in each subpicosecond slice of the bunch could be spatially separated from the radiation of electrons of the neighboring slices. Then we can either use the radiation of each slice individually or combine the radiation of all of them in a picosecond and potentially a subpicosecond pulse [5].

The separation of subpicosecond electron bunch slices is made by creating a correlation between the longitudinal coordinates of the electrons within the electron bunch and their vertical positions or angles. This would be done by exciting an RF cavity in a transverse deflection mode. The deflection couples the longitudinal and transverse motion of the electrons. In order to confine this coupling in a section of a storage ring, a second accelerating structure is needed. When placed an integer number of half betatron wavelengths downstream of the first accelerating structure, the second accelerating structure provides perfect compensation for all distortions to the longitudinal and transverse motion of the electrons made in the first accelerating structure.

One can choose to use either divergence or beam tilt to separate the radiation. In addition to the electron beam emittance and RF deflection, the natural photon beam divergence or beam size will determine the ultimate achievable bunch length for a given photon energy. For dipole sources where the photon beam divergence is large $(\sim 1 / \gamma)$, it is not practical to separate the beam using divergence. However, using the beam tilt in a dipole works well, only beginning to limit the pulse length at a few $10 \mathrm{~s}$ of $\mathrm{eV}$. For undulator sources, the photon beam divergence begins limiting the achievable pulse lengths in the few $\mathrm{keV}$. For the ALS we are initially considering using a dipole source.

The requirement that the two RF cavities need to be a half wavelength apart is easily met for the ALS. The lattice of the ALS consists of 12 sectors where each sector lattice structure is a triple bend achromat. The phase advance across a sector is naturally half a betatron wavelength. In our scheme (Figure 1), the cavities would be located in two straight sections on either side of an ALS arc sector (one of the twelve sectors). The first cavity will create a longitudinal-vertical plane correlation while the second will remove the correlation restoring the beam for the users outside the arc. In the center of the arc the beam will be maximally tilted as it passes through the central bending magnet. The light emitted from the bend magnet could be imaged onto a slit to create a short pulse RF.

The straight sections in the ALS are 5 meters long. In each straight section, the cavities will need to share the straight section space with a half length insertion device. Therefore the available space for the cavities in each straight section is less than 2.5 meters. In Table 1 we provide a list of some of the important parameters that we are considering for a picosecond source. To achieve a picosecond with the ALS parameters we are considering RF deflecting cavities with a frequency of $1.5 \mathrm{GHz}$ and $2 \mathrm{MV}$. For the parameters under consideration it is necessary to use superconducting cavities.

There are a number of challenges associated with placing RF deflecting cavities in a storage ring. The inclusion of the RF deflecting cavities should be transparent for users outside of the sector where the beam is being tilted. There should be no significant performance reduction in lifetime, beam stability and beam size.

In Table 1 we provide a list of some of the important parameters that we are considering for a picosecond source. To achieve a picosecond with the ALS parameters we are considering RF deflecting cavities with a frequency of $1.5 \mathrm{GHz}$ and $2 \mathrm{MV}$. For the parameters under consideration it is necessary to use superconducting cavities. 


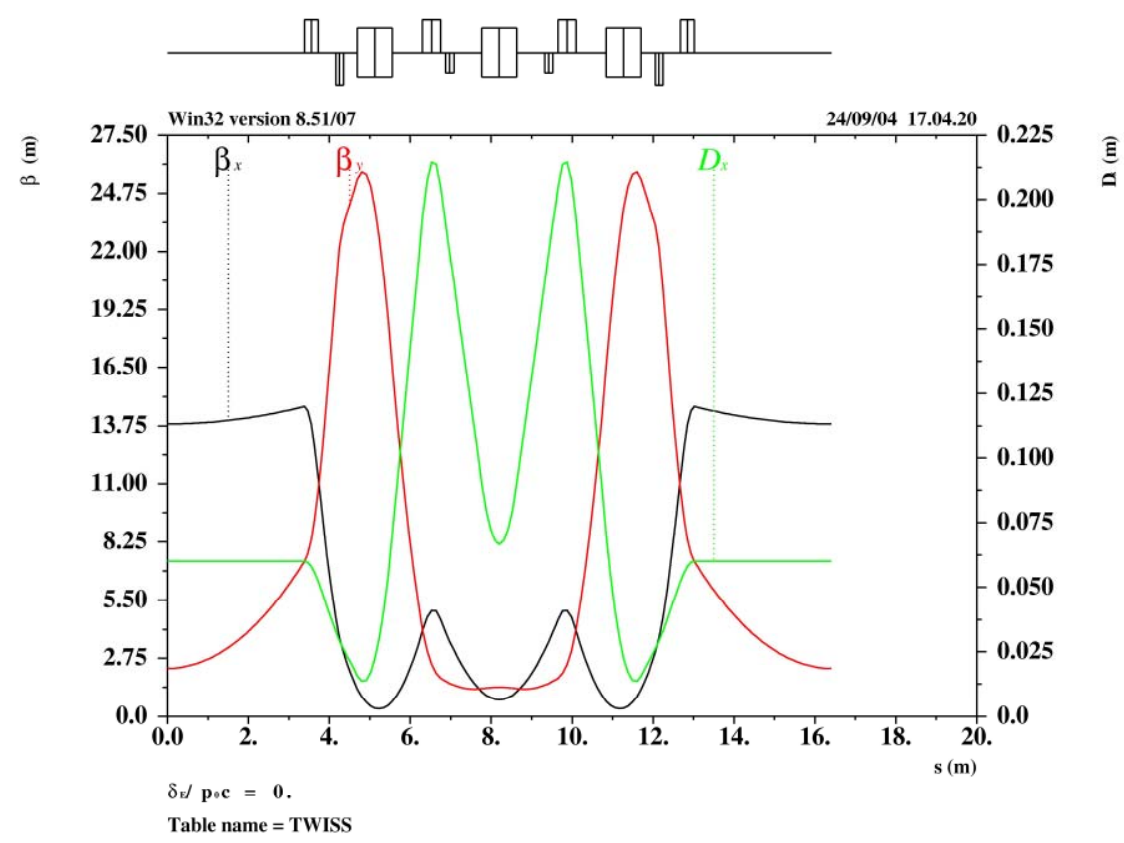

Figure 1: Arc sector of the ALS showing the location of the cavities (green boxes) the three bends (white boxes) and the vertical and longitudinal beam profile.

Table 1: Parameters of the picosecond source

\begin{tabular}{|l|l|}
\hline Parameter & Value \\
\hline RF deflecting cavity voltage & $2 \mathrm{MV}$ \\
\hline RF deflecting cavity frequency & $1.5 \mathrm{GHz}$ \\
\hline Vertical emittance & $30 \mathrm{pm} \mathrm{rad}$ \\
\hline FWHM electron bunch length & $70 \mathrm{ps}$ \\
\hline FWHM Pulse length & $0.7 \mathrm{ps}$ \\
\hline Vertical $\beta$-function at cavity & $4 \mathrm{~m}$ \\
\hline $\begin{array}{l}\text { Vertical } \beta \text {-function at central bend } \\
\text { source }\end{array}$ & $1.6 \mathrm{~m}$ \\
\hline
\end{tabular}

\section{STATUS}

We have begun looking at the feasibility of the source. There are several requirements. The first each cavity will need to occupy less than a half a straight section to leave room for an insertion device. The effect of the cavities outside of the sector must be transparent. There should be no significant impact on lifetime, injection, beam size, or beam stability. Some preliminary tracking studies indicate that for the proposed parameters we do not expect significant impact on lifetime or dynamic aperture. We have not studied the impact on the vertical emittance.

This next year we intend to further strengthen the scientific case and understand the feasibility of such a source. Particularly we will continue to investigate the beam dynamics issues and RF deflecting cavities.

\section{REFERENCES}

[1] A. Zholents and M. Zolotorev, Phys Rev Lett. 1996 Feb 5;76(6):912-915

[2] K. Harkay, et al, "Generation of Short X-Ray Pulses Using Crab Cavities at the Advanced Photon Source", these Proceedings

[3] M. Borland, et al, "Simulations of X-Ray Slicing and Compression Using Crab Cavities in the Advanced Photon Source", these Proceedings

[4] W. Guo, et al, "Generating Picosecond X-Ray Pulses with Beam Manipulation in Storage Ring Light", these proceedings

[5] A. Zholents, et al, Nuclear Instruments and Methods in Physics Research A 425 (1999) 385-389

[6] S.-B. Choe, et al, Science, 304 (2004) 420

[7] H. Stoll, et al. Applied Physics Letters, 84 (2004) 3328

[8] P. Fischer, Current Opinion in Solid State and Materials Science, 7 (2003) 173 